\title{
Psychological Stress and Antibody Response to Influenza Vaccination: When Is the Critical Period for Stress, and How Does It Get Inside the Body?
}

\author{
Gregory E. Miller, PhD, Sheldon Cohen, PhD, Sarah Pressman, MS, Anita Barkin, MSN, CRNP,
} Bruce S. Rabin, MD, PhD, and John J. Treanor, MD

\begin{abstract}
Objectives: This study attempted to determine whether stress of moderate intensity could modulate the antibody response to an influenza vaccination in healthy young adults, identify critical periods during which stress could influence antibody response, and delineate behavioral and biological pathways that might explain relations between stress and antibody. Methods: A cohort of 83 healthy young adults underwent 13 days of ambulatory monitoring before, during, and after vaccination. Four times daily, subjects reported the extent to which they felt stressed and overwhelmed and collected a saliva sample that was later used to measure cortisol. A battery of health practices (cigarette smoking, alcohol use, physical activity, sleep hygiene) was assessed daily. Antibody titers to the vaccine components were measured at baseline and at 1-month and 4-month follow-up assessments. Results and Conclusions: To the extent that they reported higher levels of stress across the monitoring period, subjects exhibited poorer antibody responses to the New Caledonia strain of the vaccine. Stress ratings on the 2 days before the vaccine and the day it was given were not associated with antibody response. However, the 10 days afterward appeared to be a window of opportunity during which stress could shape the long-term antibody response to varying degrees. With respect to potential mediating pathways, little evidence emerged in favor of cortisol secretion, alcohol consumption, physical activity, or cigarette smoking. However, analyses were consistent with a pattern in which feelings of stress and loss of sleep become locked into a feed-forward circuit that ultimately diminishes the humoral immune response. These findings may shed light on the mechanisms through which stress increase vulnerability to infectious disease. Key words: stress, daily diary, vaccine, influenza, sleep, cortisol, psychoneuroimmunology.
\end{abstract}

$\mathbf{A U C}=$ area under the curve.

\section{INTRODUCTION}

$\mathrm{E}$ vidence that psychological stress can modulate the humoral -immune response to vaccination has grown steadily in recent years (1). Prospective longitudinal studies have found that to the extent that people report high levels of stress, they produce and maintain fewer protective antibodies against pathogens including influenza, hepatitis $\mathrm{B}$, and pneumonia (2-7). These findings have potentially important theoretical and clinical implications. From a theoretical perspective, they show that stress can modulate an in vivo immune system process. This represents a significant advance for stress research with humans, which to this point has focused primarily on in vitro immune processes (8). From a clinical perspective, these findings suggest that stress may diminish the efficacy of vaccination procedures and thereby increase vulnerability to pathogens that give rise to infectious disease. Despite these important advances, much remains to be learned about the

From the Department of Psychology, University of British Columbia, Vancouver, Canada (G.E.M.); the Department of Psychology (S.C., S.P.) and Student Health Services (A.B.), Carnegie Mellon University, Pittsburgh, PA; the Department of Pathology, University of Pittsburgh School of Medicine, Pittsburgh, PA (B.S.R.); and the Department of Medicine, University of Rochester Medical Center, Rochester, NY (J.J.T.).

Address reprint requests to Gregory Miller, PhD, Department of Psychology, University of British Columbia, 2136 West Mall, Vancouver B.C., Canada V6T 1Z4. E-mail: gemiller@psych.ubc.ca

Received for publication June 14, 2003; revision received November 6, 2003.

This research was supported by a grant from the National Institute of Mental Health (MH50430) and supplemental funding from the John D. and Catherine T. MacArthur Foundation Research Network on Socioeconomic Status and Health. It was facilitated by grants from the National, Heart, Lung, and Blood Institute to the Pittsburgh Mind-Body Center (HL65111 and HL65112). Dr. Miller's efforts were supported by a Grant-in-Aid from the American Heart Association (0160367Z), a Young Investigator Award from the National Alliance for Research on Schizophrenia and Depression, and a Career Scholar Award in Clinical Science from the Michael Smith Foundation for Health Research. Dr. Cohen's participation was facilitated by a Senior Scientist Award from the National Institute of Mental Health (MH00721).

DOI: $10.1097 / 01 . p s y .0000116718 .54414 .9 \mathrm{e}$ nature of relations between psychological stress and the antibody response to vaccination.

First, the generalizability of this phenomenon remains unclear. The most robust evidence in this area is derived from studies of older adults who are providing assistance to family members suffering from dementia (2-4). These caretakers exhibit blunted antibody responses to influenza and pneumonia vaccinations compared with matched controls. The deficits in antibody response persist years after the family member has died, suggesting that chronic, severe stressors may have longterm adverse consequences for the immune system (4). Despite this intriguing pattern of findings, little is known about its generalizability. The subjects in these studies had been exposed to a stressor that was chronic, unrelenting, and severe. Would milder stressors of the kind found in daily life exert similar effects on antibody response? The subjects in these studies also were older adults with a mean age older than 65 years. The efficacy of the immune response to vaccination declines with age; older adults may therefore be especially vulnerable to stressor-induced disruptions of immunity (9). Yet it is not clear whether healthy young adults whose immune systems are intact will exhibit a similar pattern in response to stress.

Second, the kinetics of the relation between stress exposure and antibody response have not been established (1). Most studies in this area have focused on the effects of chronic, ongoing difficulties or have ignored the temporal dimension of stress altogether. Therefore, it is not clear whether there exists a critical period when stress are able to interfere with the antibody response. Knowing whether such a critical period occurs before, during, or after the vaccine is given could provide valuable mechanistic insights. The process of antibody formation is complex and unfolds in stages over a period of weeks to months. To the extent that a critical period for stress exposure can be identified, it may help shed light on which stages of antibody formation (and hence which immune processes) are responsible for the blunted humoral response. 
There would also be important clinical ramifications of these data. Psychological interventions aimed at boosting vaccine responses through stress reduction could be timed to coincide with critical periods and thereby maximize their chances of success (10). Indeed, a recent trial found that an 8-week course of mindfulness meditation boosted humoral immune responses to an influenza vaccination (11).

Finally, despite evidence that the presence of stress is associated with poorer antibody response, little is known about the mechanisms responsible for this phenomenon. Models of stress and immunity suggest that dysregulated hormone secretion or maladaptive health practices could operate as mediational pathways $(1,8)$. When demands from environment outstrip coping resources, the body's major endocrine response systems are activated, and hormones including cortisol, epinephrine, and norepinephrine are released $(12,13)$. White blood cells possess receptors for these hormones, and ligation of them results in altered patterns of cellular trafficking, proliferation and differentiation, and cytokine production $(14,15)$. Of these hormonal products, cortisol has shown the most consistent relations with antibody response in humans. Subjects who secrete the highest volumes of cortisol, either over the period of a day or in response to a stress, exhibit the poorest antibody responses to vaccination $(2,16,17)$. However, these studies have not explicitly examined whether cortisol operates as a mechanism linking stress exposure with antibody response. Health practices could also operate as a mediational pathway in this context. When people encounter difficult circumstances in their lives, they may cope with the resulting distress by altering patterns of smoking, drinking, exercise, and sleeping. Changes in each of these behaviors could modulate the immune system in a fashion that disrupts the process of antibody formation (18). Indeed, smoking, physical inactivity, and sleep debt all have been linked with diminished antibody responses to vaccination (19-21).

To begin the process of resolving these issues, this study examined antibody responses to influenza vaccination in a cohort of healthy young adults. Each subject underwent 13 days of ambulatory monitoring that covered periods before, during, and after the vaccination. Four times each day, subjects provided reports on their stress level and collected a saliva sample that was used to measure diurnal patterns of cortisol secretion. Health practices were assessed on a daily basis. This design enabled us to examine whether daily feelings of stress would relate to antibody responses over a 4-month follow-up period, identify critical periods during which feelings of stress might maximally influence antibody response to the vaccine, and explore whether cortisol secretion, health practices, or both might operate as mediational pathways.

\section{METHODS \\ Subjects}

The sample was composed of 83 first-year college students at Carnegie Mellon University. The subjects had a mean age of 18.3 years $(\mathrm{SD}=0.9)$, and slightly more than half were female $(N=46,55.4 \%)$. The majority were white $(N=55,66.3 \%)$, although a sizeable minority was of Asian descent $(N=20,24.10 \%)$. The remainder identified themselves as Hispanic $(N=2$, $2.4 \%)$, African American $(N=2,2.4 \%)$, or other $(N=4,4.8 \%)$. The subjects were enrolled in 4 cohorts during the 2000 to 2001 and 2001 to 2002 academic years, each scheduled around an influenza vaccination program offered by the university. The cohorts began the study in October $2000(N=24,28.9 \%)$, December $2000(N=30,36.1 \%)$, September $2001(N=17,20.5 \%)$, and November 2001 ( $N=12,14.5 \%)$. On completion of the study, subjects were paid $\$ 120$. The protocol was approved by the Institutional Review Board of Carnegie Mellon University.

\section{Procedures}

The subjects were recruited through advertisements in university publications, postings around campus, and announcements at social and academic functions. After contacting the project office, interested subjects received details about the study and then underwent a screening interview to determine eligibility. Subjects who were in good health, defined as having no history of chronic medical illness and no regular medication regimen other than oral contraceptives, were scheduled for a laboratory session. During this session, subjects provided written informed consent, completed a battery of psychosocial questionnaires (data not reported here), and received training in the ambulatory monitoring procedures. Subjects then began a 13-day ambulatory monitoring period (Figure 1).

To facilitate the data collection process, each subject was lent a handheld computer (ThinkPad; IBM Corp., White Plains, NY). Four times each day, the computer sounded an alarm. This signal cued subjects to answer a series of questions regarding their mood state, health practices, and physical symptoms. Their answers were recorded in the computer's memory and retrieved at the end of ambulatory monitoring period. Each day, alarms were sounded $1,4,9$, and 11 hours after the subject planned to wake in the morning. This schedule was designed on the basis of pilot studies to capture the diurnal rhythm of mood states and cortisol secretion $(22,23)$.

For a portion of the ambulatory monitoring period, days 2 to 6 of the protocol, subjects collected a saliva sample before each diary entry. Subjects collected samples by lightly chewing on cotton dental roll for 1 minute so that it was saturated with saliva (Salivette; Sartstedt Corp., Nümbrecht, Germany). The dental roll was then placed in a collection container and stored in a refrigerator until ambulatory monitoring was completed. The sample was later assayed for levels of salivary cortisol. To insure compliance with the saliva collection protocol, the handheld computer briefly flashed a numeric code each time its alarm sounded. Subjects were asked to record the code on the collection container. Those samples without proper codes were excluded from analyses.

Three days into ambulatory monitoring, subjects returned to the laboratory. A 20-ml blood sample was drawn through antecubital venipuncture and used to measure baseline antibody titers to the vaccine components. After the blood sample had been drawn, subjects were administered Fluzone vaccine by university health services personnel. In the 2000 to 2001 academic year, the Fluzone preparation contained antigens derived from the A/New Caledonia (H1N1), A/Panama (H3N2), and B/Victoria strains of influenza. The preparation for the 2001 to 2002 academic year was similar, except that antigens from the B/Victoria strain were replaced with antigens from the B/Yamanashi strain. To assess immune responses to the vaccination, subjects returned to the laboratory 1 and 4 months later. At each session, a 20-ml blood sample was

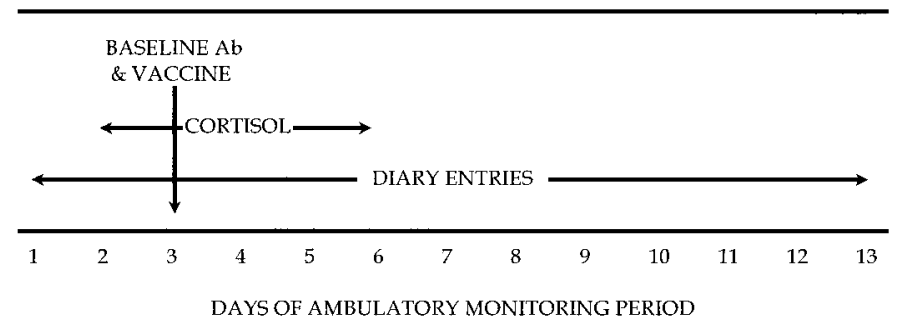

Figure 1. Timeline of ambulatory monitoring period. 
drawn through antecubital venipuncture and used to measure antibody titers to the vaccine components.

\section{Daily Ratings of Stress}

Models of the stress process suggest that distress arises when demands from the environment outstrip coping resources (12). To capture the latter phases of this process, we had subjects indicate the extent to which they felt "overwhelmed" and "stressed" at each diary entry. Response options ranged from 0 "not at all" to 4 "extremely." Because subjects' responses to these items were highly correlated (across the 52 diary entries, mean $r=0.77$, $\mathrm{SD}=0.06$ ), we later formed a composite by averaging across them. A daily stress index was then created for each day of the ambulatory monitoring period by averaging across the entries made at $1,4,9$, and 11 hours postwaking. From these data, a cumulative stress index was then created by averaging across all the days of the ambulatory monitoring period.

\section{Antibody Response to Vaccine}

Immune system responses to the influenza vaccine were assessed by quantifying antibody titers to each of the vaccine components. A standard microtiter hemagglutination inhibition protocol was used for this purpose after treatment of the sera with neuraminidase to remove nonspecific inhibitors. This methodology capitalizes on the fact that a molecule on the surface of the influenza virus, hemagglutinin, causes red blood cells to clump together or hemagglutinate. Antibody to the hemagglutinin, which is associated with protection against influenza, can be measured by the ability of sera to prevent the hemagglutination reaction. The hemagglutination inhibition titer is the reciprocal of the highest dilution at which a person's serum prevents hemagglutination. Thus, higher titer values indicate greater concentrations of antibody to a specific vaccine component. Because dilutions of serum are generally increased in geometric fashion (eg, 1:2, 1:4, 1:8), titer data are seldom distributed normally. To correct this problem, we natural log-transformed titer values. A response slope was then computed for each subject by regressing the titer level onto time since study entry. Separate response slopes were created for each vaccine component. For the purposes of interpretation, the higher the slope value, the faster the rate at which the subject produced antibody to the vaccine, and the more the subject maintained over the 4-month follow-up period.

\section{Pathways Linking Stress and Antibody Response Health Practices}

The handheld computer was used to assess health practices that might operate as pathways linking stressors with antibody response. For each of the 13 days of the ambulatory monitoring period, subjects were asked to report on smoking, alcohol consumption, and physical activity during the last diary entry of the day. Smoking was assessed by asking subjects whether they had used cigarettes, pipes, or cigars that day, and if so, how many of each they had smoked (24). Alcohol use was determined by counting the number of drinks consumed during the day. A drink was considered a bottle or can of beer, a glass of wine, or a shot of hard liquor (24). Physical activity was measured by asking subjects whether they had engaged "in an activity akin to brisk walking, jogging, bicycling, etc., long enough to work up a sweat," and if so, for how many minutes they did so (25). To assess the quantity and quality of sleep that subjects had during the monitoring period, the handheld computer presented queries about these issues during the first diary entry of each day. Sleep quantity was assessed by having subjects report the time they went to sleep the night before, the number of minutes it took them to fall asleep, and the time they woke up in the morning. Sleep quality was monitored by asking subjects to "rate their sleep quality overall last night" on a scale ranging from 1 (very poor) to 4 (very good). The sleep items were derived from the Pittsburgh Sleep Quality Index (26). To develop a cumulative index of the various health practices, we later aggregated subjects' responses across the 13 days of monitoring. This yielded cumulative indices reflecting total number of cigarettes, cigars, and pipes smoked; total number of alcoholic drinks consumed; total number of minutes spent exercising; total number of hours slept at night; and average self-reported sleep quality.

Although we do not have reliability and validity estimates for these items in a daily diary format, we have used them in previous research in which the goal was to capture longer periods of time $(22,24,27)$. They have shown excellent psychometric features in this work. The test-retest reliability coefficients were assessed over a 6-month period and were greater than 0.70 for all items except sleep quality. Subjects with chronic stressors and major depression have reported greater tobacco use, lower physical activity, and poorer sleep hygiene compared with healthy controls. In turn, these health practices have been linked with in vitro measures of immune response, such as reduced natural killer cell cytotoxicity and poorer mitogen-stimulated lymphocyte proliferation, and impaired resistance to upper respiratory infection.

\section{Cortisol Secretion}

After they were returned to the laboratory, Salivettes were centrifuged for 5 minutes at $3000 \mathrm{rpm}$ until a clear, low-viscosity supernatant emerged. The supernatants were then aspirated and frozen at $-70^{\circ} \mathrm{C}$ until the end of the study, at which time they were shipped on dry ice to the Institute of Experimental Psychology II at the University of Duesseldorf. Cortisol assays were performed in duplicate using a time-resolved fluorescence immunoassay with a cortisol-biotin conjugate as a tracer (28-30). This assay has a sensitivity of $0.43 \mathrm{nmol} / \mathrm{l}$. The intra-assay and interassay coefficients of variation were less than $10 \%$ and less than $12 \%$, respectively.

After the cortisol values had been log-10 transformed, each day's data were used to create an area under the curve (AUC) index. The AUC reflects the total volume of cortisol secretion over the period of the day. It was computed using the trapezoidal method, such that higher values reflect greater cortisol release. Because AUC values were highly correlated across the 5 days of sample collection (mean interday $r=0.69, \mathrm{SD}=0.10$ ), we developed a cumulative index of cortisol secretion by aggregating across the ambulatory monitoring period. To insure that we had reliable estimates of AUC, daily values were excluded from analyses if they were based on fewer than 3 samples or if a morning value was not available for the calculation. Fewer than $10 \%$ of the daily values ( 41 of $415,9.9 \%$ ) had to be excluded for these reasons.

\section{Data Cleaning}

Because the sampling schedule we used was specially designed to capture diurnal fluctuations in mood states and cortisol secretion, we felt that it was important to monitor subjects' compliance with ambulatory monitoring carefully, and to exclude any diary entries that did not conform to the protocol's requirements. The handheld computers' capacity to time-stamp and datestamp each diary entry facilitated this process greatly. On an a priori basis, we chose to define compliance as making a diary entry within 60 minutes of target in either direction. When this definition was applied, a total of 3756 of the 4316 diary entries (87\%) met our criteria for compliance. Only these values were used in the data analyses reported here.

\section{RESULTS}

\section{Previous Exposure to Vaccine Components}

Preliminary analyses explored subjects' previous exposure to the vaccine components. The geometric mean of prevaccine titer values was $18.75(\mathrm{SD}=242.27)$ for New Caledonia, $51.51(\mathrm{SD}=306.87)$ for Panama, $19.58(\mathrm{SD}=150.41)$ for Victoria, and $3.91(\mathrm{SD}=23.79)$ for Yamanashi. In clinical settings, previous exposure is usually inferred when titer values are 4 or greater (31). By this standard, $80.7 \%$ of subjects had been exposed to New Caledonia $(N=67), 96.4 \%$ to Panama $(N=80), 83.3 \%$ to Victoria $(N=40)$, and $37.9 \%$ to Yamanashi $(N=11)$. A handful of subjects exhibited maximal titers (1024) before they had been given the vaccine. This was the case for 5 subjects with New Caledonia and 6 subjects with Panama. The analyses presented here include these subjects even though they could not show any increase over time 
as a result of the assay's upper limits of detection. However, separate analyses excluding these subjects yielded an identical pattern of findings.

\section{Antibody Response to Vaccine}

Nearly all of the subjects exhibited an adequate immune response to the vaccine. Virologists evaluate vaccine responses according to 1 of 2 standards: whether the subject displays a 4-fold increase in antibody titers after the vaccine, and whether the subject reaches a level of antibody considered to be protective in the face of pathogen exposure (usually $\geq 40 ; 31$ ). By the 4 -fold standard, $97.6 \%$ of subjects displayed an adequate response to New Caledonia $(N=81), 85.5 \%$ to Panama $(N=71), 57.4 \%$ to Victoria $(N=31)$, and $89.7 \%$ to Yamanashi $(N=26)$. A similar pattern emerged when the protection standard was used to evaluate responses. Of the subjects, $94.0 \%$ displayed an adequate response to New Caledonia $(N=78), 88.8 \%$ to Panama $(N=73), 85.2 \%$ to Victoria $(N=46)$, and $72.4 \%$ to Yamanashi $(N=21)$. Given that our subjects were young adults in good health, these high rates of vaccine response are not surprising.

\section{Does Stress Predict Antibody Response?}

The first wave of statistical analyses examined whether daily reports of stress were associated with antibody response to the vaccine in our sample of healthy young adults. This analysis focused on cumulative experiences of stress during the ambulatory monitoring period. To examine this question, a series of regression equations was constructed in which antibody response slopes were predicted from a) baseline antibody titer values, b) a series of control variables that included age, race, gender, and cohort, and c) a cumulative stress index formed by averaging ratings across the 13-day monitoring period. Separate equations were constructed for each vaccine component.

Table 1 displays the results of this analysis for the New Caledonia strain. Beyond the effects of baseline antibody and the control variables, the cumulative stress index was negatively associated with antibody response slopes. That is, to the

TABLE 1. Cumulative Stressor Ratings Predict Antibody Response to Influenza Vaccination

\begin{tabular}{lccccc}
\hline \multicolumn{1}{c}{ Predictor } & B & SE B & $\beta$ & $t$ & $p$ \\
\hline $\begin{array}{l}\text { Step 1 } \\
\quad \text { Baseline antibody }\end{array}$ & -.08 & .02 & -.47 & -4.67 & $<.01$ \\
$\quad \begin{array}{l}\text { Step 2 } \\
\text { Age }\end{array}$ & -.09 & .06 & -.16 & -1.41 & $<.16$ \\
Race & -.02 & .02 & -.12 & -1.16 & $<.25$ \\
$\quad$ Gender & -.06 & .05 & -.12 & -1.08 & $<.28$ \\
$\quad$ Cohort & -.05 & .03 & -.20 & -1.80 & $<.08$ \\
$\begin{array}{l}\text { Step 3 } \\
\quad \text { Cumulative stress } \\
\text { index }\end{array}$ & -.04 & .02 & -.24 & -2.44 & $<.02$ \\
\hline
\end{tabular}

${ }^{a}$ For step 1 of equation cumulative $\mathrm{R}^{2}=.23, \Delta \mathrm{R}^{2}=.23, p<.001$; for step 2 , cumulative $\mathrm{R}^{2}=.31, \Delta \mathrm{R}^{2}=.09, p<.08$; and for step 3, cumulative $\mathrm{R}^{2}$ $=.36, \Delta \mathrm{R}^{2}=.05, p<.02$. extent that subjects reported higher levels of stress across the monitoring period, the slower they produced antibody to New Caledonia, and the less they maintained over the 4-month follow-up period.

To facilitate interpretation of these findings, we stratified subjects into low-stress, medium-stress, and high-stress groups based on their cumulative stress index being less than, within, or greater than $1 \mathrm{SD}$ from the sample mean. Figure 2 displays antibody response slopes for New Caledonia as a function of this grouping. As is evident from the figure, response slopes differed significantly among the groups $(F[2,76]=4.12, p<.03)$. Subjects in the high-stress group had significantly flatter slopes than those in the medium-stress $(F[1,64]=4.54, p<.04)$ and low-stress groups $(F[1,29]=$ $8.15, p<.02)$, who did not differ reliably from each other $(F[1,67]=0.46$, NS $)$. The average cumulative score for subjects in the high-stress group was 5.12 ( $\mathrm{SD}=0.90$ ), indicating that they perceived themselves to be moderately stressed and overwhelmed during the monitoring period.

To get a better sense of stress-related differences at each assessment, we plotted logged titer values at baseline, 1 month, and 4 months as a function of groupings on the cumulative stress index (Figure 3 ). The findings indicate that the groups began the study with similar antibody levels $(F[1,82]=0.23$, NS $)$ but showed differing patterns of response to the vaccine over the follow-up period. At both 1 month and 4 months, subjects in the high-stress group had significantly fewer antibodies compared with subjects in the medium-stress $(F[1,64]=7.43, p<.01)$ and low-stress groups $(F[1,29]=5.20, p<.04)$, whose values were nearly identical to each other $(F[1,68]=0.49, \mathrm{NS})$. The differences in antibody titer values between subjects in the high-stress group and the other groups ranged from $12 \%$ to $17 \%$.

Despite these positive findings for New Caledonia, the stress index did not reliably predict antibody response slopes to the Panama, Victoria, or Yamanashi strains ( $p$ values $=.97$, $.40, .12$, respectively).

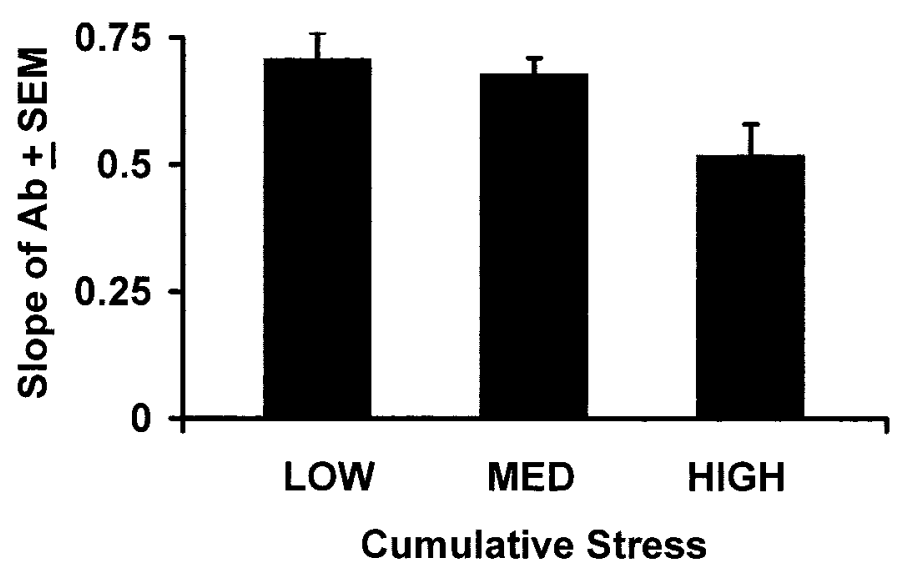

Figure 2. Antibody response slope to influenza vaccine as a function of cumulative stress ratings. Subjects are stratified into low-stress, mediumstress, and high-stress groups based on their ratings being less than, within, or greater than $1 \mathrm{SD}$ from the sample mean. Response to New Caledonia strain is shown. 


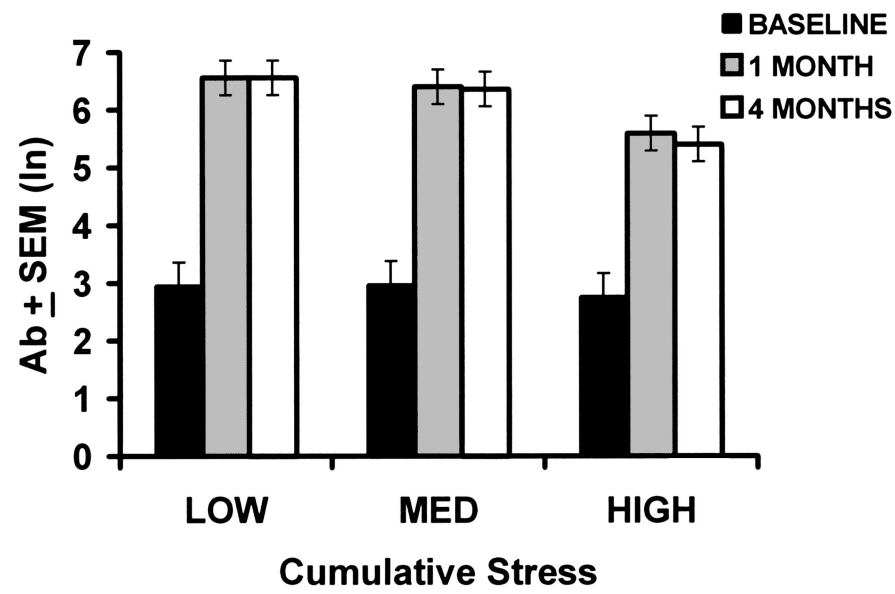

Figure 3. Antibody titer values at baseline, 1-month, and 4-month assessments as a function of cumulative stress ratings. Subjects are stratified into low-stress, medium-stress, and high-stress groups based on their ratings being less than, within, or greater than $1 \mathrm{SD}$ from the sample mean. Response to New Caledonia strain is shown.

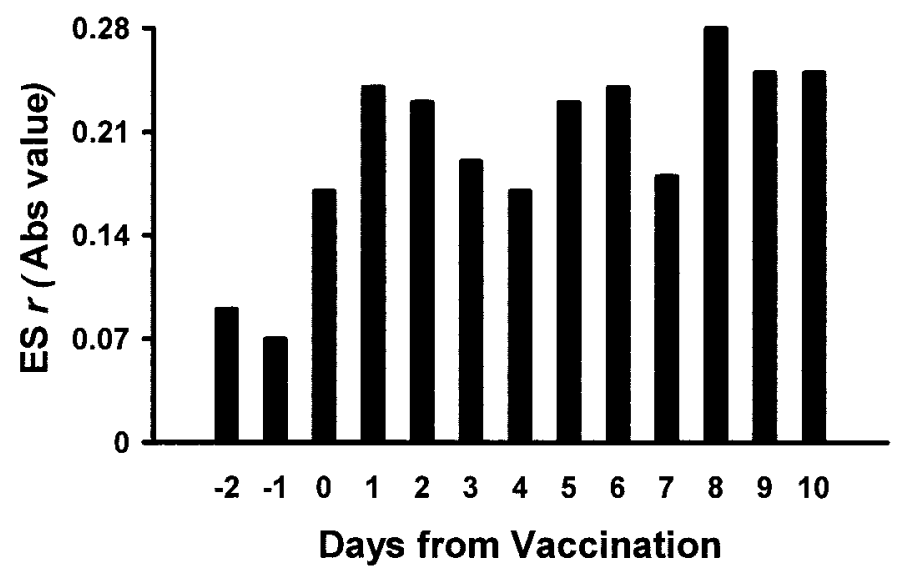

Figure 4. Effect sizes relating stress ratings to antibody response for each day of ambulatory monitoring. Note that all effect sizes are negatively signed (ie, higher stress relates to lower antibody), but they are displayed as absolute values for the sake of presentation.

\section{When Is the Critical Period?}

With findings suggesting that cumulative stress diminishes antibody response to the New Caledonia strain, we next sought to identify critical periods of time around the vaccination during which stressors might be especially influential. To do so, we constructed a series of hierarchical regression equations in which the New Caledonia antibody response slope was predicted from baseline antibody titer values, the control variables, and 1 of the daily stress indices.

Table 2 displays the results of these analyses. The findings indicate that stress levels during the prevaccine period were not associated with antibody response over the follow-up period. The same was true of stress on the day of the vaccine. Stress levels on the 3 days postvaccine were inversely associated with antibody response. That is, to the extent that subjects reported higher levels of stress on these days, the slower they produced antibody to New Caledonia, and the less they maintained over the follow-up period. The effect sizes for
TABLE 2. Daily Ratings of Stress and Slope of Antibody Response to Influenza Vaccination

\begin{tabular}{cccccc}
\hline $\begin{array}{c}\text { Days from } \\
\text { Vaccination }\end{array}$ & $\mathrm{B}$ & $\mathrm{SE} \mathrm{B}$ & $\beta$ & $\Delta \mathrm{R}^{2}$ & $\mathrm{ES} r$ \\
\hline-2 & -.01 & .02 & -.09 & .01 & -.09 \\
-1 & -.00 & .01 & -.02 & .00 & -.02 \\
0 & -.02 & .01 & -.17 & .03 & -.17 \\
1 & -.03 & .01 & -.26 & $.06^{* *}$ & -.24 \\
2 & -.03 & .01 & -.24 & $.05^{* *}$ & -.23 \\
3 & -.03 & .01 & -.20 & $.04^{*}$ & -.19 \\
4 & -.02 & .01 & -.18 & .03 & -.17 \\
5 & -.03 & .01 & -.24 & $.05^{* *}$ & -.23 \\
6 & -.03 & .01 & -.24 & $.05^{* *}$ & -.24 \\
7 & -.02 & .01 & -.19 & .03 & -.18 \\
8 & -.04 & .01 & -.30 & $.08^{* * *}$ & -.28 \\
9 & -.03 & .01 & -.26 & $.06^{*}$ & -.25 \\
10 & -.03 & .01 & -.25 & $.06^{*}$ & -.25
\end{tabular}

$\Delta \mathrm{R}^{2}=$ change in variance accounted for by stressor ratings; ES $r=$ effect size associated with each day's stressor rating as correlation. ${ }^{*} p<.06$; ${ }^{* *} p<.05$; $* * * p<.01$.

these days ranged from $r=-0.19$ to -0.24 . By Cohen's standards, these are small to medium effects. Although stress 4 days after the vaccine was not associated with the antibody response slope, days 5 and 6 showed reliable inverse relations with it, and the effect sizes were again small to medium ( $r$ values $=-0.23$ to -0.24 ). The strongest relations between daily stress and antibody response occurred 8 to 10 days after the vaccine. On these days, there were reliable inverse relations between stress ratings and response slope, and the effect sizes were $r=-0.25$ to -0.28 . Figure 4 displays the absolute value of the effect sizes relating stress ratings to antibody response for each day of ambulatory monitoring.

Because stress ratings were highly correlated across days of ambulatory monitoring, we next sought to identify days that might independently predict antibody response slope to the New Caledonia strain. To do so, we constructed another hierarchical regression equation in which slope was predicted from baseline antibody titers, the 4 control variables, and all 13 of the daily stress ratings. In this analysis, the only stress rating to emerge as an independent and significant predictor of response slope was made 8 days after the vaccine $(B=-0.08$, $\mathrm{SE} \mathrm{B}=0.04, \beta=-0.66, p<.04$, effect size $r=-0.22$ ). As was the case in earlier analyses, higher levels of stress on this day predicted a poorer antibody response. For all of the other days, $p$ values for stress ratings exceeded .16 .

\section{What Are the Pathways Linking Stress and Antibody Response?}

Having found that cumulative stress diminishes antibody response to the New Caledonia strain, we next examined whether cortisol secretion, health practices, or both might operate as mediating pathways. Another series of regression equations was constructed in which the candidate pathway was entered on the step before stress. Following standard procedures (32), evidence for mediation was inferred when the 
presence of a candidate pathway substantially reduced the variance that stress explained in antibody response. As a way to confirm the results of this analysis, we also performed Sobel tests in cases in which findings were consistent with mediation. This test examines whether an indirect pathway linking stress, the candidate pathway, and antibody response is statistically reliable. Because the original formulation of the Sobel test lacks statistical power, we used a version with modified critical values as suggested by MacKinnon et al. (33). At the outset, we should note that these methods cannot provide a strong test of whether the mediators operate in a causal fashion. Such a test would require an experimental manipulation. What they can provide, however, is an indication of whether each mechanism relates to cumulative stress and antibody response in a way that is consistent with a mediational hypothesis.

\section{Cortisol Secretion}

Analyses indicated that cumulative stress was unrelated to volume of cortisol secretion across the 5-day saliva collection period $(r=0.03, p=.81)$. The volume of cortisol secretion, in turn, was unrelated to antibody response $(r=0.02, p=$ .87). When cortisol secretion volume was entered into the regression equations described previously, it did not reduce the amount of variance that cumulative stress explained in antibody response. Together, these findings suggest that cortisol was not operating as a pathway through which cumulative stress diminished antibody response over the 4-month follow-up period. ${ }^{1}$

\section{Health Practices}

Cumulative stress ratings were unrelated to minutes of intense physical activity, number of alcoholic drinks, and self-rated sleep quality across the ambulatory monitoring pe$\operatorname{riod}(r=-0.04, p=.70 ; r=0.09, p=.43 ; r=-0.13, p=$ .24 , respectively). Not surprisingly, entering these health practices into regression equations did not reduce the amount of variance that cumulative stress explained in antibody response. Subjects who reported higher levels of stress tended to smoke more cigarettes over the monitoring period $(r=0.23$, $p<.04)$. However, smoking volume was unrelated to antibody response slopes $(r=0.12, p=.29)$, and controlling for it did not attenuate the effects of cumulative stress.

\footnotetext{
${ }^{1}$ An insightful reviewer suggested 2 alternative hypotheses regarding stress, cortisol, and antibody response. First, it could be that we did not detect relations between stress ratings and cortisol secretion because their measurement periods had only partial overlap. To match the timing of stress and cortisol assessments more closely, we later created a cumulative stress index spanning just the 5 days when saliva was collected. However, this index was unrelated to volume of cortisol secretion $(r=.02, p=.89)$, suggesting that this was not a likely explanation for our null findings. Second, it could be that the diurnal rhythm of cortisol secretion, rather than the volume of output, is responsible for stress-antibody relations. To examine this hypothesis, we used each day's cortisol data to compute slopes reflecting the diurnal rhythm of secretion, and then averaged them over the ambulatory monitoring period. Analyses indicated that cumulative stress was unrelated to diurnal rhythm $(r=.01, p=.99)$. This measure was also unrelated to antibody response $(r=$ $.12, p=.32$ ), suggesting that altered diurnal cortisol rhythms were not responsible for the poorer antibody response among subjects high in stress.
}

Analyses indicated that subjects who reported higher levels of cumulative stress also tended to have fewer hours of sleep during ambulatory monitoring $(r=-0.33, p<.01)$. Hours of sleep was marginally associated with antibody response $(r=$ $-0.20, p=.08$ ), such that subjects with fewer hours had reduced slope values. When hours of sleep during ambulatory monitoring was entered into the regression equations described earlier, it reduced the amount of variance that cumulative stress accounted for in antibody response slope from $5.4 \%$ to $2.2 \%$. This represents a $59.3 \%$ reduction in variance accounted for by stress. The effect of cumulative stress also become nonsignificant under these conditions $(\mathrm{B}=-0.02$, $\mathrm{SE} \mathrm{B}=0.02, \beta=-0.17, p<.12$, effect size $r=-0.15$ ). These findings suggest that sleep was operating as a mediational pathway. To confirm these results, we performed a Sobel test examining whether the indirect pathway linking cumulative stress, total sleep, and antibody response was statistically significant. It yielded a $z$ value of 1.74 , which is significant at $p<.05$, according to the modified degrees of freedom suggested by MacKinnon et al. (33). These findings are consistent with the view that cumulative stress diminished antibody responses to the New Caledonia strain by reducing the amount of sleep that subjects were able to get over the ambulatory monitoring period.

\section{What Is the Nature of Stress-Sleep Relations?}

Because stress and sleep data were collected over the same period, the analyses presented here cannot elucidate the direction of their relationship. Feelings of stress could diminish sleep quantity, as we suggest, but a lack of sleep could also foster stress. To sort out these competing possibilities, we took advantage of our diary reports and examined prospective relations between these processes. These analyses were performed using hierarchical linear modeling (34). The withinperson relations between stress and sleep were estimated using restricted maximum likelihood with robust standard errors. A first-order autoregressive covariance matrix was specified to control for the autocorrelation generated by data collected on subsequent days from the same person (35). Models were estimated using HLM v5.04 (Scientific Software International; 36).

The first model examined whether ratings of stress across the day would predict the amount of sleep that subjects reported that evening. To ensure that the relation between these processes was not inflated by what occurred the previous evening, each model included a covariate representing the hours of sleep obtained the night before. The results of this analysis indicated that ratings of stress were inversely associated with hours of sleep $(b=-0.19, \mathrm{SE}=0.07, t[82]=$ $-2.95, p<.01)$. That is, to the extent that subjects rated their stress as higher during the day, they tended to get fewer hours of sleep that evening, even when the quantity of sleep from the night before was controlled. These findings are consistent with the hypothesis that stress diminishes sleep.

The second model examined the opposite hypothesis: that lack of sleep at night fosters greater stress the next day. As in 
the model described earlier, the previous day's stress level was included as a covariate. The results of this analysis indicated that sleep hours were inversely related to stress levels $(b=$ $-0.04, \mathrm{SE}=0.01, t[82]=-2.52, p<.02)$. To the extent that subjects reported fewer hours of sleep on a given night, they tended to have higher ratings of stress on the next day. These findings are consistent with the view that sleep loss fosters feelings of stress. However, given that both of these diarybased analyses yielded positive findings, the most appropriate conclusion is that bidirectional relations between these processes exist in our data.

\section{DISCUSSION}

The first goal of this study was to determine whether daily reports of stress were associated with antibody response to an influenza vaccine in healthy young adults. Findings indicated that to the extent that they reported higher levels of cumulative stress, subjects produced antibody to New Caledonia at a slower rate and maintained less antibody over the 4-month follow-up period. This effect was most evident among subjects in the high-stress group. Although these subjects perceived themselves to be only moderately stressed during ambulatory monitoring, their antibody response was reduced by $12 \%$ to $17 \%$ at 1 month and 4 months after the vaccine compared with the lower-stress groups. It remains unclear whether these response disparities have clinical significance. Of the subjects in this high-stress group, $75 \%$ had what virologists consider adequate protection at the follow-up assessments, corresponding to a titer value greater than 40 . Also, stressor ratings were not associated with antibody to the other vaccine components. Overall, these findings suggest that selfrated stress of moderate intensity is capable of blunting antibody responses in healthy young adults, but in a fairly modest fashion.

The study also examined whether there is a critical period when stressors interfere with the antibody response. Results indicated that stressor ratings during the period before vaccination and the day it was given did not reliably predict the extent of antibody production. During the week-long period after the vaccine was administered, daily stressor ratings were inversely associated with long-term antibody response. For the most part, these relations were statistically significant, with small to medium effects ranging from $r=-0.18$ to -0.24 . The strongest relations between stressor ratings and antibody response emerged during the latter portion of ambulatory monitoring. The $r=-0.28$ effect size for day 8 , for instance, was the largest we found, and the only daily rating that independently predicted antibody response. Overall, this pattern of findings suggests that although the capacity of stressors to influence antibody response varies across days, there is probably no single critical period to which its effects are restricted. Instead, there appears to be a broader window of opportunity for stressors that opens the day after the vaccine is given and lasts for at least 10 days afterward. Because a variety of immune system processes are unfolding during this time, we cannot specify which of them is being modulated in a way that leads to blunted antibody responses over the long term. However, the timing of the alterations suggests that the latter stages of the antibody production process are being affected more than the earlier stages. Thus, stress-related modulation of cytokine production by activated $\mathrm{T}$ cells may account for the lower antibody response to the New Caledonia strain $(1,15)$.

The study also sought to identify mediational pathways linking cumulative stressor ratings with patterns of antibody response. Although previous research has suggested that cortisol might be such a pathway $(2,16,17)$, we found no evidence that it was linked with stressor ratings or vaccine response. It is not clear why our findings diverge from previous research in this respect. The 5 days of ambulatory monitoring yielded a more reliable estimate of cortisol secretion than previous studies had, so it is difficult to imagine that measurement strategy alone would be a viable explanation for these disparate findings. It is possible that we missed the critical period for cortisol by measuring it only until 3 days after the vaccine was administered. Indeed, it was after saliva collection had ended that stressors had their strongest relations with antibody response, suggesting that cortisol secretion later in the monitoring period might have been an important pathway linking stressors and antibody. With regard to health practices, there was little evidence that alcohol consumption, physical activity, and cigarette smoking were responsible for the inverse relations between cumulative stress and antibody response.

Sleep quantity did emerge as a potential mediator. To the extent that they reported higher levels of cumulative stress, subjects tended to get fewer hours of sleep across the monitoring period, which in turn was associated with a flatter antibody response slope. Two different statistical strategies for assessing mediation provided evidence consistent with the hypothesis that cumulative stress diminished antibody responses by reducing the amount of sleep that subjects were able to get. Follow-up analyses of the diary reports yielded a more complex pattern of findings, however, and suggested that feelings of stress and loss of sleep were locked into a feed-forward circuit in which each promoted the other. Although it is not clear whether this cycle begins with stress or sleep, our findings suggest that the result is a blunted immune response to a routine vaccination. In this respect, our findings converge with previous research suggesting relations among stressors, sleep patterns, and immunity $(37,38)$. They also converge with a compelling series of experimental demonstrations that sleep deprivation can modify immune processes such as natural killer cell cytotoxicity, inflammatory cytokine production, and antibody responses to vaccination $(21,39,40)$.

Several potential limitations of this study must be highlighted. First, although our findings represent an important empirical advance, they were restricted to the New Caledonia strain of the vaccine. It is unclear why a similar pattern of results did not emerge for the other vaccine components. Although subjects' average response slopes were greatest for 
New Caledonia, the variances were similar across all 4 strains, suggesting that a restriction of range explanation is probably inadequate in this context. It is possible that we had limited statistical power to detect stress-related alterations in the antibody response to $\mathrm{B} /$ Victoria and $\mathrm{B} / \mathrm{Y}$ amanashi, because only a subset of subjects $(65 \%$ and $35 \%$, respectively) received these components as part of their Fluzone vaccination. We are not aware of structural or functional differences between the vaccine components that would render them differentially responsive to stress. However, we are inclined to believe that any strain difference in stressor responsivity arises early in the process of antibody formation (eg, during antigen processing and presentation by dendritic cells), because the latter phases of the humoral response are likely to be similar regardless of vaccine component (15). Future research that can provide insights into these puzzling findings will be especially valuable, because a growing number of studies in this area are finding that the effects of stress are restricted to A-strains of the influenza vaccine $(2,41)$. Second, our measure of vaccine response was limited to antibody titers as indexed by hemagglutination inhibition. However, for a vaccine to confer protection against infectious pathogens, it needs to stimulate a broad array of immune system processes. Future studies can extend research in this area by assessing processes such as antibody binding capacity, the activation of complement, vaccine-specific cytokine production by helper $\mathrm{T}$ lymphocytes, and T-lymphocyte cytotoxic capacity against vaccine components. Third, the study's ambulatory monitoring period lasted only for a period of 13 days. With additional monitoring, we would have been able to determine how long the vulnerability window remains open - that is, how far into the process of antibody production stressors remain an influential determinant of the longer-term humoral response. Future studies with longer ambulatory monitoring periods, and extended collection of hormonal products such as cortisol, would be quite useful in this regard. Finally, the study's focus on daily stressors in healthy young adults limits its generalizability such that we cannot be certain whether its findings would extend to medically vulnerable populations who are grappling with more challenging circumstances.

Despite the study's methodological shortcomings, it extends research in this area by showing that stress of moderate intensity are capable of blunting antibody responses to vaccination in healthy young adults with intact immune systems; identifying the 10 days after the vaccine as a window of opportunity during which stress can shape the long-term antibody response to varying degrees; and highlighting a pattern in which feelings of stress and loss of sleep become locked into a feed-forward circuit that ultimately diminishes the humoral immune response to a routine vaccination. With further research efforts in the directions specified, this line of work may shed light on the mechanisms through which stress contribute to morbidity and mortality in the context of infectious disease.
The authors extend their gratitude to Elena Balestreire, Ellen Conser, Ruomei Liang, Leona Middleton, and Kristen Weinzierl for their help with data collection and management; to Dr. Clemens Kirschbaum for conducting the salivary cortisol assays; and to Rosalyn Battaglia for performing the hemagglutination inhibition protocol.

\section{REFERENCES}

1. Cohen S, Miller GE, Rabin BS. Psychological stress and antibody response to immunization: a critical review of the human literature. Psychosom Med 2001;63:7-18.

2. Vedhara K, Cox NKM, Wilcock GK, Perks P, Hunt M, Anderson S, Lightman SL, Shanks NM. Chronic stress in elderly caregivers of demential patients and antibody response to influenza vaccination. Lancet 1999;353:627-31.

3. Kiecolt-Glaser JK, Glaser R, Gravenstein S, Malarkey WB, Sheridan JF. Chronic stress alters the immune response to influenza virus vaccine in older adults. Proc Natl Acad Sci U S A 1996;93:3043-7.

4. Glaser R, Kiecolt-Glaser JK, Malarkey WB, Sheridan JF. The influence on psychological stress on the immune response to vaccines. Ann N Y Acad Sci 1998;840:649-55.

5. Petrie KJ, Booth RJ, Pennebaker JW, Davison KP, Thomas MG. Disclosure of trauma and immune response to a hepatitis B vaccination program. J Consult Clin Psychol 1995;63:787-92.

6. Jabaaij L, Grosheide PM, Heijtink RA, Duivenvoorden HJ, Ballieux RE, Vingerhoets AJJM. Influence of perceived psychological stress and distress on antibody response to low dose rDNA hepatitis B vaccine. J Psychosom Res 1993;37:361-9.

7. Glaser R, Sheridan JF, Malarkey WB, MacCallum RC, Kiecolt-Glaser JK. Chronic stress modulates the immune response to a pneumococcal pneumonia vaccine. Psychosom Med 2000;62:804-7.

8. Segerstrom SC, Miller GE. Psychological stress and the immune system: a meta-analytic study of 30 years of inquiry. Under review. 2003.

9. Solomon GF, Benton D. Psychoneuroimmunologic aspects of aging. In: Glaser R, Kiecolt-Glaser JK, editors. Handbook of human stress and immunity. New York: Academic Press; 1994. p. 341-63.

10. Miller GE, Cohen S. Psychological interventions and the immune system: a meta-analytic review and critique. Health Psychol 2001;20:47-63.

11. Davidson RJ, Kabat-Zinn J, Schumacher J, Rosenkranz M, Muller D, Santorelli SF, Urbanowski F, Harrington A, Bonus K, Sheridan JF. Alterations in brain and immune function produced by mindfulness meditation. Psychosom Med 2003;65:564-70.

12. Lazarus RS, Folkman S. Stress, appraisal, and coping. New York: Springer; 1984.

13. Baum A, Cohen L, Hall M. Control and intrusive memories as possible determinants of chronic stress. Psychosom Med 1993;55:274-86.

14. Blalock JE. The syntax of neuroendocrine-immune communication. Immunol Today 1994;15:504-11.

15. Rabin BS. Stress, immune function, and health: the connection. New York: John Wiley \& Sons; 1999.

16. Boyce WT, Adams S, Tschann JM, Cohen F, Wara D, Gunnar MR. Adrenocortical and behavioral predictors of immune responses to starting school. Pediatr Res 1995;38:1009-17.

17. Burns VE, Ring C, Drayson M, Carroll D. Cortisol and cardiovascular reactions to mental stress and antibody status following hepatitis $B$ vaccination: a preliminary study. Psychophysiology 2002;39:361-8.

18. Kiecolt-Glaser JK, Glaser R. Methodological issues in behavioral immunology research with humans. Brain Behav Immun 1988;2:67-78.

19. Horowitz MM, Ershler WB, McKinney WP, Battiola RJ. Duration of immunity after hepatitis B vaccination: efficacy of low-dose booster vaccine. Ann Intern Med 1988;108:185-9.

20. Kohut ML, Cooper MM, Nickolaus MS, Russell DR, Cunnick JE. Exercise and psychosocial factors modulate immunity to influenza vaccine in elderly individuals. J Gerontol Biol Sci Med Sci 2002;57:M557-62.

21. Spiegel K, Sheridan JF, Van Cauter E. Effect of sleep deprivation on response to immunization. JAMA 2002;288:1471-2.

22. Miller GE, Cohen S, Ritchey AK. Chronic psychological stress and the regulation of pro-inflammatory cytokines: a glucocorticoid resistance model. Health Psychol 2002;21:531-41.

23. MacArthur Foundation Network on SES and Health. Salivary cortisol measurement. San Francisco, CA: MacArthur Foundation; 2000.

24. Cohen S, Doyle WJ, Skoner DP, Rabin BS, Gwaltney JM Jr. Social ties and susceptibility to the common cold. JAMA 1997;277:1940-4.

25. Paffenbarger RS, Blair SN, Lee I, Hyde RT. Measurement of physical 
activity to assess health effects in a free-living population. Med Sci Sports Exerc 1993;25:60-70.

26. Buysse DJ, Reynolds CF, Monk TH, Berman SR, Kupfer DJ. The Pittsburgh Sleep Quality Index: a new instrument for psychiatric practice and research. Psychiatry Res 1989;28:193-213.

27. Miller GE, Cohen S, Herbert TB. Pathways linking major depression and immunity in ambulatory female patients. Psychosom Med 1999;61: $850-60$.

28. Kirschbaum C, Kudielka BM, Gaab J, Schommer NC, Hellhammer DH. Impact of gender, menstrual cycle phase, and oral contraceptives on the activity of the hypothalamic-pituitary-adrenal axis. Psychosom Med 1999;61:154-62.

29. Kirschbaum C, Hellhammer D. Salivary cortisol in psychobiological research: an overview. Neuropsychobiology 1989;22:150-69.

30. Pruessner JC, Hellhammer DH, Kirschbaum C. Burnout, perceived stress, and cortisol responses to awakening. Psychosom Med 1999;61:197-204.

31. Cox JH, deSouza M, Ratto-Kim S, Ferrari G, Weinhold KJ, Birx DL. Cellular immune assays for evaluation of vaccine efficacy in developing countries. In: Rose NR, Hamilton RG, Detrick B, editors. Manual of clinical laboratory immunology. 6th ed. Washington, DC: ASM Press; 2002. p. 301-17.

32. Stone AA. Selected methodological concepts: mediation and moderation, individual differences, aggregation strategies, and variability of replicates. In: Schneiderman N, McCabe P, Baum A, editors. Stress and disease processes: perspectives in behavioral medicine. Hillsdale, NJ: Lawrence Erlbaum Associates; 1992. p. 55-71.
33. MacKinnon DP, Lockwood CM, Hoffman JM, West SG, Sheets V. A comparison of methods to test mediation and other intervening variable effects. Psychol Methods 2002;7:83-104.

34. Bryk AS, Raudenbush SW. Hierarchical linear models. Newbury Park, CA: Sage; 1992.

35. Schwartz JE, Stone AA. Strategies for analyzing ecological momentary assessment data. Health Psychol 1998;17:6-16.

36. Raudenbush SW, Bryk AS, Congdon RT. HLM version 5.04. Chicago, IL: Scientific Software International; 2001.

37. Hall M, Baum A, Buysse DJ, Prigerson HG, Kupfer DJ, Reynolds CF. Sleep as a mediator of the stress-immune relationship. Psychosom Med 1998;60:48-51.

38. Ironson G, Wynings C, Schneiderman N, Baum A, Rodriguez MS, Greenwood D, Benight C, Antoni MH, LaPerriere A, Huang H, Klimas NG, Fletcher MA. Posttraumatic stress symptoms, intrusive thoughts, loss, and immune function after Hurricane Andrew. Psychosom Med 1997;59:128-41.

39. Irwin MR, Mascovich A, Gillin JC, Willoughby R, Pike J, Smith TL. Partial sleep deprivation reduces natural killer cell activity in humans. Psychosom Med 1994;56:493-8.

40. Irwin MR. Effects of sleep and sleep loss on immunity and cytokines. Brain Behav Immun 2002;16:503-12.

41. Stetler CA, Chen E, Miller GE. Written disclosure of experiences with racial discrimination and antibody response to an influenza vaccine. Under review. 2003. 